\title{
UM ESTUDO SOBRE ANÁLISE, REPRESENTAÇÃO E DETECÇÃO DE INTENÇÕES DE CRIMINOSOS EM POSTAGENS EM MÍDIA SOCIAL
}

\author{
Ricardo Resende de Mendonça ${ }^{1}$, Ferrucio de Franco Rosa ${ }^{2}$ e Rodrigo Bonacin ${ }^{1,2}$ \\ ${ }^{1}$ UNIFACCAMP, Campo Limpo Paulista, SP, Brasil \\ ${ }^{2}$ CTI Renato Archer, Campinas, SP, Brasil
}

\begin{abstract}
RESUMO
As mídias sociais se transformaram em um instrumento de comunicação entre criminosos, para planejamento e execução de crimes, bem como para recrutar novos membros. Eles utilizam diferentes estratégias de comunicação, tais como linguagens cifradas e gírias restritas a grupos ou facções para burlar investigações. A pesquisa por ferramentas e técnicas para apoiar a atividade investigativa e o processo de prevenção de crimes são de extrema importância; particularmente, a análise e detecção de intenções relacionadas a crimes. Os aspectos tecnológicos, humanos e sociais relacionados a este problema, torna-o um campo rico de estudo, envolvendo a interação entre IHC (Interação Humano-Computador) com diversas áreas de pesquisa. Pesquisas ligadas às áreas de segurança de informação, linguística, aprendizagem de máquina e processamento de linguagem natural, têm contribuído para o avanço na análise e detecção de intenções em mídia social. Este artigo apresenta uma revisão quasi-sistemática da literatura sobre análise, representação e detecção de intenções de criminosos em postagens em mídia social. 27 estudos foram analisados de acordo com as abordagens utilizadas (ex.: técnicas de aprendizagem de máquina), bem como seus fundamentos em aspectos linguísticos, ontologias, semiótica e teoria dos atos da fala. Os resultados apontam avanços na solução do problema e questões de pesquisas em aberto para a área de IHC e relacionadas.
\end{abstract}

\section{PALAVRAS-CHAVE}

Crime, Redes Sociais, Sistemas Web, Linguagem, IHC, Segurança da Informação

\section{INTRODUÇÃO}

Crimes são planejados, relatados ou executados com o auxílio de redes sociais. O uso das redes sociais por criminosos visa a construção de comunidades virtuais, mobilização, treinamento online, disseminação de seus serviços ou ideais, recrutamento de novos integrantes, financiamento, etc. Criminosos se comunicam nas redes utilizando um linguajar próprio (cifrado) que visa a dificultar a compreensão por pessoas externas ao grupo. Uma avaliação de 119 crimes cometidos por criminosos que atuaram sozinhos demonstrou que $35 \%$ deles interagiram com comunidades criminosas online durante o planejamento do crime e $45 \%$ aprenderam técnicas para execução do crime em recursos online (Gill et al., 2017). A comunicação é restrita a determinadas pessoas, regiões e a um período de tempo (Mendonça et al., 2019). Devido ao grande volume de dados não estruturados disponíveis nas redes sociais, analisar postagens de maneira automatizada é um desafio (Idrees, Alam, \& Agarwal, 2018). A extração do conhecimento das redes sociais é prejudicada, pois os dados gerados pelos usuários muitas vezes são não estruturados e contém um alto grau de informalidade; textos informais estão muito presentes na comunicação diária entre os usuários (Wu, Morstatter, \& Liu, 2018). A linguagem natural utilizada em redes sociais é curta e informal, o que por si só já representa um desafio computacional (Wu et al., 2018). A utilização de gírias incorpora uma complexidade ainda maior na elaboração de algoritmos computacionais eficientes. Um mapeamento das gírias faz-se necessário, uma vez que estas alteram o significado real das palavras com a intenção de ludibriar investigações. Neste contexto, este artigo tem como principal objetivo responder a seguinte questão de pesquisa: "Como avaliar e representar computacionalmente a intenção de criminosos em postagens escritas em linguagem natural com a utilização de gírias?". Além de analisar e classificar os estudos de acordo com as abordagens técnicas empregadas, este artigo também discute os fundamentos em aspectos linguísticos, ontologias, semiótica 
(Peirce, 1994) e teoria dos atos da fala (Austin, 1975) (Searle, 1969). Os principais objetivos desta revisão são: (i) identificar as técnicas e teorias utilizadas, os aspectos positivos e limitações dos estudos existentes; e (ii) apontar lacunas na literatura e desafios de pesquisa, atuais e futuros. O restante deste artigo está organizado da seguinte maneira: a Seção 2 apresenta outras revisões sobre temas relacionados à questão principal; a Seção 3 descreve a metodologia empregada para realizar a revisão e análise dos artigos; a Seção 4 detalha os resultados obtidos categorizados pelas técnicas utilizadas; e a Seção 5 apresenta uma discussão sobre os resultados obtidos e as conclusões.

\section{TRABALHOS RELACIONADOS}

Ao todo, 6 revisões sistemáticas foram identificadas no decorrer da análise dos estudos coletados. A Tabela 1 (Apêndice I) apresenta uma síntese dos trabalhos relacionados; os trabalhos foram enquadrados de acordo com seus objetivos e aplicações. Uma análise sintética desses trabalhos é apresentada a seguir.

Fortuna \& Nunes (2018) apresentam uma revisão sobre a detecção automática de discursos de ódio em texto escritos em linguagem natural. Segundo os autores, houve um aumento de estudos nesta área, onde, $42,1 \%$ dos estudos realizados utilizaram a rede social Twitter, com predominância do idioma inglês. Observa-se também um aumento na utilização de técnicas de aprendizagem de máquina. A revisão não analisou fundamentos como os aspectos linguísticos, ontologias, semiótica e teoria dos atos da fala. Salawu, He, \& Lumsden (2017) buscam elucidar quais técnicas computacionais são mais relevantes na detecção automática de cyberbullying, comportamento antissocial e assédio. O estudo evidenciou uma busca por aprimoramento nas técnicas para análise de textos em linguagem natural. A revisão destaca que determinar o estado emocional de uma vítima de cyberbullying é uma área de pesquisa ainda em aberto. A adoção de algoritmos de aprendizado supervisionado é a abordagem mais presente na área de detecção de cyberbullying. Os autores destacam que mesmo sendo revelado um alto número de pesquisas na área em questão, as redes sociais ainda fazem o uso de recursos que dependem da interação humana para gerar alertas de cyberbullying, especialmente nos aspectos relacionados a imagens e vídeos. O foco dos autores está nas técnicas computacionais e a revisão não abrangeu, por exemplo, o planejamento de crimes por meio da Internet (abordado neste artigo). Ravi \& Ravi (2015) buscaram identificar avanços e tendências em análise de sentimentos e mineração de opiniões. SVM (Support Vector Machine) e abordagens baseadas em dicionários estão presentes em 56,14\% dos estudos avaliados. A baixa utilização de ontologias foi identificada nos estudos avaliados. Segundo os autores, o uso de ontologias permitiu reduzir o problema de imprecisão na análise de sentimento. A revisão foca em análise de sentimentos e opiniões que podem, ou não, estar ligados a crimes; não abrangendo, portanto, estudos que focam aspectos especificamente relacionados a atividades criminosas. Kumar \& Sachdeva (2019) buscam coletar, explorar e compreender a detecção de cyberbullying, bem como identificar lacunas. As redes sociais possuem características que tornam a detecção de atividades de cyberbullying computacionalmente difícil; dentre essas características se destaca o uso de gírias e a escrita informal. Segundo os autores, SVM é a técnica mais utilizada na análise e detecção de cyberbullying. Rosa et al. (2019) reforçam a tendência (39\%) da utilização de SVM para detecção de cyberbullying. Os autores destacam o fraco desempenho na detecção automática de cyberbullying, devido à necessidade de aprimorar o pré-processamento. Omar, Fred, \& Swaib (2018) visam elucidar a detecção de fraudes. As técnicas mais utilizadas foram Redes Neurais, Árvore de decisão e SVM. Os autores destacam que a ausência de padronização na utilização ou criação de datasets transforma a tarefa de validação e aprimoramento dos métodos uma tarefa complexa e por muitas vezes infactível.

A revisão apresentada neste artigo se diferencia das demais revisões nos seguintes aspectos: (1) o foco desta revisão está na análise, representação e detecção de intenções de criminosos em postagens em mídia social, enquanto as revisões existentes focam aspectos como a detecção de cyberbullying (mais frequente), análise de sentimento, discursos de ódio, fraudes, comportamento antissocial e assédio; (2) a maioria das revisões analisadas foca em aspectos técnicos, não abrangendo uma discussão mais ampla sobre fundamentos de aspectos linguísticos, ontologias, semiótica e teoria dos atos da fala; e, (3) os estudos não analisam como os estudos tratam o uso de linguagens cifradas por criminosos nas redes sociais. 


\section{METODOLOGIA DA REVISÃO}

A revisão quasi-sistemática da literatura apresentada neste artigo teve como base metodológica o guia apresentado em (Kitchenham, 2004). Este estudo tem como principal objetivo responder a seguinte questão de pesquisa: "Quais são as abordagens para avaliar e representar computacionalmente a intenção de criminosos em postagens escritas em linguagem natural com a utilização de gírias?". Uma pesquisa exploratória preliminar baseada na questão de pesquisa foi realizada com o objetivo de levantar insumos necessários à pesquisa, resultando na definição dos parâmetros da pesquisa, no período de abrangência da busca, nas bases científicas e palavras-chave a serem utilizadas, e na área de busca nos artigos. O período de busca (2014 a 2019) se deve ao fato de ser um tema relativamente recente, bem como se espera relatar os avanços nos últimos anos. A seguinte string de busca foi utilizada (adaptada a sintaxe de cada base): "(ontology OR thesaurus OR taxonomy OR vocabulary) AND (intention OR semiotics OR "speech acts") $A N D$ (crime OR criminal)". A execução da busca nas bases científicas considerou todos os artigos retornados, com exceção da base Google Scholar. Em função da abrangência desta base (indexadora de outras bases), foram considerados os 100 primeiros artigos por ordem de relevância. Assim, a busca inicial obteve um total de 1.876 artigos, sendo 289 artigos de Springer Link, 346 de IEEE Xplore, 763 de Science Direct, 378 de ACM Digital Library e 100 de Google Scholar. Os critérios de inclusão e exclusão foram definidos por três pesquisadores, sendo dois doutores e um especialista da área da computação, em um processo iterativo de leitura de artigos (na busca exploratória) e proposição de critérios até atingir consenso. Os critérios estão detalhados na Tabela 2 (Apêndice II). 24 artigos foram excluídos por duplicidade nos resultados das bases científicas. Os artigos remanescentes foram submetidos aos critérios de inclusão e exclusão. A primeira avaliação considerou o título, resumo e palavras-chave. Os 42 artigos categorizados como trabalhos com possibilidade de aderência ao tema da pesquisa foram avaliados em sua totalidade perante os critérios. Três pesquisadores analisaram os artigos selecionados e elaboraram a lista final em consenso após discussões. Durante essa avaliação, 8 estudos foram identificados como não aderentes e 6 trabalhos são revisões de literatura relacionadas ao tema e foram descritas na Seção 2.

\section{RESULTADOS DA REVISÃO}

Esta seção apresenta uma análise sintética dos 27 estudos selecionados. A Subseção 4.1 apresenta soluções baseadas em Dicionário Léxico e em ontologias para a análise de intenções, enquanto a subseção 4.2 apresenta soluções baseadas em aprendizagem de máquina. A Tabela 3 (Apêndice III) apresenta uma síntese dos trabalhos analisados.

\subsection{Soluções Baseadas em Dicionário Léxico e em Ontologias}

Teodorescu \& Saharia (2015), Teh, Cheng, \& Chee (2018) e Xiaomei, Jing, \& Jianpei (2018) abordam o uso de dicionários léxicos, bem como suas limitações para avaliação de sentimentos. Teodorescu \& Saharia, (2015) classificam cada palavra de forma manual e fazem uso de XML. Segundo os autores, uma gíria pode conter inúmeros significados, ou até mesmo a sua utilização pode ser completamente diferente em culturas distintas. Segundo Waseem, Thorne, \& Bingel (2018), os trabalhos de análise de sentimentos não consideram adequadamente a influência de questões sociais, geográficas e culturais; tal fator prejudica sua eficácia. Segundo Teh et al. (2018), a utilização de um dicionário léxico não é suficiente para detectar a presença de discurso de ódio em textos escritos em linguagem natural, uma vez que a evolução do vocabulário deve ser considerada. $\mathrm{O}$ estudo propõe a categorização dos termos de ódio em 8 categorias distintas. Em (Xiaomei et al., 2018), utiliza-se um dicionário léxico que correlaciona uma palavra com um sentimento da roda de emoções de Plutchik. Após estabelecer essa correlação, hashtags são avaliadas para identificar a que evento se refere a postagem. Hagen et al. (2015) ontologias, técnicas linguísticas e avaliação de emoticons são usados para classificar o sentimento no momento da coleta dos registros. Uma primeira ontologia faz a distinção entre ataques, defesas, atacante e objetivo. O resultado desta fase é avaliado por um analisador de sentimentos que determina se o conteúdo é inofensivo ou uma ameaça. Segundo os autores, para domínios estáticos uma ontologia bem definida é suficiente. No entanto, para ambientes dinâmicos faz-se necessária uma constante atualização, com a possível mudança ou eliminação de conceitos já validados anteriormente. 


\subsection{Soluções Baseadas em Aprendizagem de Máquina e Mistas}

Dentre os 27 estudos avaliados, 22 exploram técnicas de aprendizagem de máquina, como elemento central em propostas para identificação de intenções e sentimentos (ex.: ódio e depressão) em textos escritos em linguagem natural. As três principais técnicas de aprendizagem de máquina identificadas foram Redes Neurais, SVM e Naive Bayes; alguns desses estudos são considerados mistos, pois fazem uso de ontologias em conjunto com aprendizagem de máquina. A seguir apresenta-se uma síntese dos trabalhos.

García-Díaz et al. (2018) definem o processo de extração de sentimento como "opinion mining", que consiste na utilização de processamento de linguagem natural e linguística computacional. O processo de mineração de dados se dá em 5 etapas, a saber: seleção, pré-processamento, transformação, mineração de dados e interpretação. No pré-processamento dos tweets os nomes dos usuários e citações a usuários foram convertidas para um identificador para garantir o anonimato A quarta etapa é responsável por gerar a saída que deverá ser trabalhada por meio da técnica de Naive Bayes. Segundo os autores, o trabalho antes da utilização de aprendizagem de máquina permitiu uma melhora significativa nos resultados. Losada \& Crestani (2016), propõem um processo de detecção de depressão em linguagem natural que consiste de quatro etapas, a saber: seleção da origem dos textos; extração dos dados; predição, que consiste na análise do histórico das mensagens de um determinado usuário na plataforma; e por fim, a classificação dos textos por meio do algoritmo de Logistic Regression.

Em (Anzovino, Fersini, \& Rosso, 2018), busca-se a detecção de misoginia em mensagens postadas no Twitter. Uma categorização dos tipos de misoginia é proposta no estudo, utilizando aprendizagem de máquina e PLN (Processamento de Linguagem Natural) como estratégia para classificação dos tweets como misóginos. Waseem et al. (2018) usam técnicas de aprendizagem de máquina com múltiplas tarefas de processamento intermediárias visando o aprimoramento da assertividade. Assim como em (García-Díaz et al., 2018), esse processo também apresentou melhora nos resultados, porém, com perda de desempenho.

Agarwal \& Sureka (2017) buscam detectar racismo em postagens na rede social Tumblr. Um dicionário léxico foi criado e inspecionado manualmente. Uma análise linguística foi realizada por meio de duas APIs (Alchemy Document Sentiment e IBM Watson Tone Analyzer). Cinco categorias de sentimentos foram propostas: alegria, medo, tristeza, raiva e desgosto. Um experimento fez uso de três técnicas de aprendizagem de máquina: Random forest, Decision Tree e Naive Bayes. Os resultados evidenciaram a superioridade da técnica de Random Forest em relação às outras. Um método baseado em deep neural network, combinando Convolutional Neural Networks (CNN) e Gated Recurrent Netwotks (GRN) é proposto em (Zhang, Robinson, \& Tepper, 2018). Seu desempenho foi superior a SVM, SVM+ e CNN.

Vários fatores dificultam o reconhecimento de sarcasmo, tais como a falta contexto, uso de gírias e o uso de perguntas retóricas. Em (Ghosh, Fabbri, \& Muresan, 2018) busca-se analisar a detecção de sarcasmo em redes sociais e fóruns de discussão, por meio de SVM e de Long Short-Term Memory (LSTM); LSTM obteve melhores resultados do que SVM. Em Justo et al. (2014) busca-se identificar sarcasmo e maldade em comentários realizados em redes sociais. Para realizar a classificação, duas técnicas foram utilizadas (classificação baseada em regras e Naive Bayes). Apesar de inicialmente parecerem similares, sarcasmo e maldade são distintos na forma de detecção, uma vez que para a detecção de sarcasmo é necessário dominar a área de conhecimento e incluir características (ex.: contexto), para melhor avaliação. Considerando isso, os autores adicionaram outras técnicas para detecção de sarcasmo, a saber: categorias léxicas usando $n$-Grams e detecção de sentimento por meio da semântica.

Em (Appling, Briscoe, \& Hutto, 2015), os autores indicam o uso de técnicas de linguística para detectar farsa em textos, além do uso de um modelo discriminatório. Uma das técnicas linguísticas utilizadas é a quantidade de palavras usadas em um determinado texto para afirmação de algo. A quantidade maior que a média daquele usuário indica a possibilidade de farsa. Esse tipo de técnica é prejudicado por depender do conhecimento prévio das postagens realizadas pelo usuário. Hu \& Wang, (2016) propõem um modelo matemático para utilização em conjunto com Naive Bayes. Um comparativo alterando o algoritmo para Decision Tree evidencia a superioridade de Naive Bayes.

Segundo Dhouioui \& Akaichi (2016), uma combinação de técnicas para aprimoramento da detecção de predadores sexuais faz-se necessária. Os autores propõem a utilização de mineração de textos para classificar conversas; essa classificação ocorre por meio de três dicionários léxicos e extração de características comportamentais. Foi feita uma comparação entre SVM e Naive Bayes, sendo que o primeiro apresentou superioridade de desempenho. Três dicionários léxicos foram desenvolvidos, a saber: (i) Emoticons; (ii) Contrações da língua inglesa; e (iii) Termos utilizados normalmente na comunicação via SMS. 
Em (Barreira, Pinheiro, \& Furtado, 2017) os autores propõem a utilização de Semantic Role Labeling (SRL) para análise forense de mensagens de textos extraídos de dispositivos móveis. Segundo os autores, a associação de técnicas linguísticas e aprendizagem de máquina apresenta melhor precisão do que quando as técnicas são utilizadas de maneira isolada. Levitan et al. (2015) visam a detecção de fraudes em discursos em vídeos. A transcrição do áudio é necessária para aplicação da técnica proposta, que faz uso de Random Forest e Bagging. Segundo os autores a adoção de um dicionário léxico permitirá um aprimoramento dos resultados.

Raisi \& Huang (2017) buscam a detecção de cyberbullying por meio de técnicas de aprendizagem de máquina. Após determinar um par de usuários, é realizada uma análise de vários tweets desse par para determinar se existe a caracterização de bullying. Essa abordagem se torna restrita a ambientes em que se possui acesso ao histórico de mensagens trocadas entre os envolvidos. Pandey et al. (2019) categorizam intenções com base em técnicas de aprendizagem de máquina (linear model of logistic regression e CNN) apoiadas por recursos de semântica distribucional. Três categorias de intenções foram formuladas: acusação, confirmação e sensacionalismo.

Escalante et al. (2017) buscam detectar em postagens de redes sociais fraudes ou agressões, antes destas acontecerem. Segundo os autores, a maioria das soluções se aplica à detecção de eventos que já ocorreram. O trabalho recomenda a utilização de perfil e sub-perfil para auxiliar o processo de detecção. O perfil é definido por meio de dois vetores de mesma dimensão contendo palavras (3-grams) e suas classificações. A adoção de sub-perfis permite a avaliação de múltiplas classes, pois leva em consideração a diversidade de domínios. Na abordagem proposta, o processo de detecção ocorre por meio da análise de documentos já categorizados por meio de algoritmos de aprendizagem de máquina.

Mundra et al. (2017) propõem uma técnica baseada em redes neurais para classificação de expressões idiomáticas (presentes em microblogs como o Twitter). Em (Chen, Santoso, Lee, \& Wang, 2016), os autores avaliaram a adoção de Latent Dirichlet Allocation e Collaborative representation classifier. Segundo os autores, um sistema de detecção de intenções de crimes deve utilizar mais de uma técnica de aprendizagem de máquina. Sharma \& Sarma (2017) usam características dos sons emitidos na comunicação por voz para detecção de humor e dialeto do usuário. Parâmetros de Fourier são usados para apoiar a detecção e posteriormente a categorização de humor e dialeto. A técnica SVM foi utilizada na categorização. Os autores também avaliaram a adoção de RNN (Recurrent Neural Network) e DTDNN (Distributed Time Delay Neural Network), porém em nenhum dos cenários ocorreu superioridade a SVM.

Aghababaei \& Makrehchi (2017) propõem um modelo preditivo que cruza dados públicos do FBI em alguns estados dos Estados Unidos com os tweets de usuários mais ativos e com contas registradas há mais tempo. O modelo faz o treinamento e as predições de maneira automática. O objetivo principal do estudo é conseguir prever a ocorrência de crimes em uma determinada área em função das publicações do Twitter.

Park \& Rayz (2019) usam técnicas de aprendizagem de máquina para criação de uma ontologia. Segundo os autores, o conhecimento semântico adquirido por meio do uso da ontologia permite uma melhor compreensão e categorização dos ataques de phishing (tentativa de obtenção de informação pessoalmente identificável). Suárez-Serrato et al. (2018) apresentam um estudo com o objetivo de compreender a diferença de comportamento e intenção de usuários e socialbots no Twitter. A extração de conhecimento foi realizada por meio da técnica de TF-IDF (Term Frequency Inverse Document Frequency); após a fase de extração, a análise de sentimento foi realizada por meio do software LabMT.

\section{DISCUSSÃO E CONCLUSÃO}

Observa-se que a avaliação e representação de intenções dos usuários em textos escritos em linguagem natural é muito escassa. Contudo, foram identificadas técnicas e aperfeiçoamentos na área de categorização de emoções e sentimentos que abordam intenções indiretamente. As propostas avaliadas fazem uso de técnicas de processamento de linguagem natural (Anzovino et al., 2018), (Appling et al., 2015), (Barreira et al., 2017), (Hagen et al., 2015), (Hu \& Wang, 2016), (Justo et al., 2014), (Lundquist, Zhang, \& Ouksel, 2015), (Maynard, Bontcheva, \& Augenstein, 2016), mineração de dados e análise da semântica (García-Díaz et al., 2018). Neste sentido, 9 estudos (Anzovino et al., 2018), (Appling et al., 2015), (Barreira et al., 2017), (García-Díaz et al., 2018), (Hagen et al., 2015), (Hu \& Wang, 2016), (Justo et al., 2014), (Lundquist et al., 2015), (Maynard et al., 2016) apresentaram melhores índices de assertividade na categorização de sentimentos. Mesmo apresentando redução de desempenho, o benefício da adoção de técnicas 
complementares supre o custo computacional. Classificadores probabilísticos são comprovadamente confiáveis, porém, com forte dependência ao tamanho da base de treinamento (García-Díaz et al., 2018). A abordagem proposta apresenta ganhos na realização de pós-processamento, onde se obtém melhora na precisão dos classificadores aplicando conhecimento específico de domínio. Assim, esses classificadores não são adequados para análise de dados em tempo real, tornando a realização do pré-processamento uma tarefa imprescindível para obtenção de melhor precisão.

A adoção de múltiplas técnicas para detecção de intenções e sentimentos apresenta-se como uma prática promissora. Anzovino et al. (2018) obtiveram melhores resultados com a utilização de Token N-Grams em conjunto com SVM. Appling et al. (2015), destacam os benefícios da adoção de uma estratégia-múltipla (execução de PLN em conjunto com aprendizagem de máquina). A adoção de ontologias em conjunto com outras técnicas para análise de sentimentos ou intenções ainda se apresenta como uma área de estudo em aberto e requer contínuos esforços para seu avanço. Park \& Rayz (2019) destacam que a utilização exclusiva de um dicionário léxico apresentou vulnerabilidades, pois essa característica não está presente quando ontologias são empregadas para análise da semântica em textos escritos em linguagem natural. Segundo Lundquist et al. (2015), a utilização de PLN em conjunto com ontologias apresentaram bons resultados, alcançando $86 \%$ de precisão.

Embora emoções e sentimentos estejam fortemente ligados a intenções, estudos com foco específico em intenções são raros, particularmente aqueles com fundamentação teórica consistente sobre o entendimento de intenções. Por exemplo, apenas um estudo (Hu \& Wang, 2016) faz uso explicitamente da teoria dos atos da fala (Austin, 1975) como referencial teórico. A perspectiva em que as palavras são usadas para realizar coisas tem muito a contribuir, não só no processo de detecção, mas principalmente em estabelecer o que se deseja detectar. Por exemplo, ilocuções (atos de falar ou escrever que constituem ações) podem resultar em efeitos pragmáticos diferentes, dependendo da interpretação das intenções do falante. Assim, um modelo de classificação de ilocuções pode contribuir na definição do que se deseja detectar ao falarmos sobre "intenções dos usuários", como, por exemplo, cometer crimes. Embora semiótica fosse uma palavra-chave da busca, não foram obtidos estudos que analisem postagens ligadas a intenções de cometer crimes. A semiótica nos fornece uma vasta base teórica e metodológica para entender o uso e interpretação de signos em sistemas computacionais (Andersen, 2001). Do ponto de vista da semiótica, as pessoas se comunicam por meio de múltiplas mídias compartilhando signos. Langford (1938) define a pragmática como um ramo de estudo que tenta entender a relação entre signos e pessoas, sendo fundamental para compreender a intenção do locutor. Nessa perspectiva, é importante entender como os signos influenciaram o processo de comunicação para detectar intenções de criminosos em postagens em redes sociais. A análise, representação e detecção de intenções de criminosos em postagens em mídia social carece de um arcabouço teórico e metodológico mais amplo. Este arcabouço envolve o estudo da interação do humano com (e mediado por) artefatos tecnológicos, os aspetos linguísticos e o comportamento do usuário, bem como técnicas avançadas de aprendizagem de máquina.

Este artigo apresentou uma revisão quasi-sistemática da literatura sobre a análise, representação e detecção de intenções de criminosos em postagens em mídia social. A abordagem empregada nesta revisão da literatura permitiu a verificação e análise de tendências, bem como abordagens tecnológicas adotadas ao longo dos últimos cinco anos. Este estudo se diferencia dos demais ao focar na análise e representação computacional sobre intenções criminais presentes em postagens em linguagem natural com a utilização de gírias e linguagem cifradas. Em um universo de 1852 artigos inicialmente recuperados, 27 trabalhos foram criteriosamente selecionados, classificados e sintetizados de modo a representar o estado-da-arte das abordagens para avaliar e representar computacionalmente a intenção de criminosos em postagens escritas em linguagem natural com a utilização de gírias. Foram apresentadas as técnicas e teorias utilizadas, os aspectos positivos e limitações dos estudos, bem como apontadas lacunas na literatura e desafios de pesquisa, atuais e futuros. Os estudos foram analisados e sintetizados de acordo com as abordagens e técnicas utilizadas, bem como seus fundamentos em aspectos linguísticos, ontologias, semiótica e teoria dos atos da fala. Observou-se que a avaliação e representação de intenções dos usuários em textos escritos em linguagem natural é muito escassa. Pesquisas multidisciplinares relacionadas à segurança de informação, linguística, aprendizagem de máquina e processamento de linguagem natural, também contribuem para o avanço na análise e detecção de intenções em mídias sociais. Os resultados apontam avanços na solução do problema e questões de pesquisas em aberto para a área de IHC (Interação Humano-Computador) e relacionadas. 
Além de apresentar uma revisão de literatura abrangente sobre análise, representação e detecção de intenções de criminosos em postagens em mídia social, este trabalho também contribuiu para uma proposta de formalização conceitual de expressões criminais por meio de uma ontologia (Mendonça et al., 2019).

\section{REFERÊNCIAS}

Agarwal, S., \& Sureka, A. (2017). But i did not mean it! - Intent classification of racist posts on tumblr. Proceedings - 2016 European Intelligence and Security Informatics Conference, EISIC 2016, $124-127$. https://doi.org/10.1109/EISIC.2016.032

Aghababaei, S., \& Makrehchi, M. (2016). Mining Social Media Content for Crime Prediction. In 2016 IEEE/WIC/ACM International Conference on Web Intelligence (WI) (pp. 526-531). IEEE. https://doi.org/10.1109/WI.2016.0089

Aghababaei, S., \& Makrehchi, M. (2017). Mining Social Media Content for Crime Prediction. Proceedings - 2016 IEEE/WIC/ACM International Conference on Web Intelligence, WI 2016, 526-531. https://doi.org/10.1109/WI.2016.0089

Andersen, P. B. (2001). What Semiotics can and cannot do for HCI. Knowledge-Based Systems, 14(8), $419-424$. https://doi.org/10.1016/S0950-7051(01)00134-4

Anzovino, M., Fersini, E., \& Rosso, P. (2018). Automatic Identification and Classification of Misogynistic Language on Twitter. In E. Métais, F. Meziane, M. Saraee, V. Sugumaran, \& S. Vadera (Eds.) (Vol. 7934, pp. 57-64). Berlin, Heidelberg: Springer Berlin Heidelberg. https://doi.org/10.1007/978-3-319-91947-8_6

Appling, D. S., Briscoe, E. J., \& Hutto, C. J. (2015). Discriminative Models for Predicting Deception Strategies. In Proceedings of the 24th International Conference on World Wide Web - WWW'15 Companion (pp. 947-952). New York, New York, USA: ACM Press. https://doi.org/10.1145/2740908.2742575

Austin, J. L. (1975). How To Do Things With Words. Oxford University Press. https://doi.org/10.1093/acprof:oso/9780198245537.001.0001

Barreira, R., Pinheiro, V., \& Furtado, V. (2017). A framework for digital forensics analysis based on semantic role labeling. In 2017 IEEE International Conference on Intelligence and Security Informatics: Security and Big Data, ISI 2017 (pp. 66-71). https://doi.org/10.1109/ISI.2017.8004876

Chen, S. H., Santoso, A., Lee, Y. S., \& Wang, J. C. (2016). Latent dirichlet allocation based blog analysis for criminal intention detection system. In Proceedings - International Carnahan Conference on Security Technology (Vol. 2015-Janua, pp. 73-76). https://doi.org/10.1109/CCST.2015.7389660

de Mendonça, R. R., de Franco Rosa, F., Theophilo Costa, A. C., Bonacin, R., \& Jino, M. (2019). OntoCexp: A Proposal for Conceptual Formalization of Criminal Expressions. In 16th International Conference on Information Technology-New Generations (ITNG 2019) (pp. 43-48). https://doi.org/10.1007/978-3-030-14070-0_7

Dhouioui, Z., \& Akaichi, J. (2016). Privacy Protection Protocol in Social Networks Based on Sexual Predators Detection. In Proceedings of the International Conference on Internet of Things and Cloud Computing (pp. 1-6). New York, NY, USA: ACM. https://doi.org/10.1145/2896387.2896448

Escalante, H. J., Villatoro-Tello, E., Garza, S. E., López-Monroy, A. P., Montes-y-Gómez, M., \& Villaseñor-Pineda, L. (2017). Early detection of deception and aggressiveness using profile-based representations. Expert Systems with Applications, 89, 99-111. https://doi.org/10.1016/j.eswa.2017.07.040

Fortuna, P., \& Nunes, S. (2018). A Survey on Automatic Detection of Hate Speech in Text. ACM Computing Surveys, 51(4), 1-30. https://doi.org/10.1145/3232676

García-Díaz, V., Espada, J. P., Crespo, R. G., Pelayo G-Bustelo, B. C., \& Cueva Lovelle, J. M. (2018). An approach to improve the accuracy of probabilistic classifiers for decision support systems in sentiment analysis. Applied Soft Computing Journal, 67, 822-833. https://doi.org/10.1016/j.asoc.2017.05.038

Ghosh, D., Fabbri, A. R., \& Muresan, S. (2018). Sarcasm Analysis Using Conversation Context. Computational Linguistics, 44(4), 755-792. https://doi.org/10.1162/coli_a_00336

Gill, P., Corner, E., Conway, M., Thornton, A., Bloom, M., \& Horgan, J. (2017). Terrorist Use of the Internet by the Numbers: Quantifying Behaviors, Patterns, and Processes. Criminology and Public Policy, 16(1), 99-117. https://doi.org/10.1111/1745-9133.12249

Hagen, L., Harrison, T. M., Uzuner, Ö., Fake, T., Lamanna, D., \& Kotfila, C. (2015). Introducing textual analysis tools for policy informatics. In Proceedings of the 16th Annual International Conference on Digital Government Research - dg.o '15 (pp. 10-19). New York, New York, USA: ACM Press. https://doi.org/10.1145/2757401.2757421

Hu, Y., \& Wang, S. (2016). Research on Crime Degree of Internet Speech Based on Machine Learning and Dictionary. In Proceedings - 2016 3rd International Conference on Information Science and Control Engineering, ICISCE 2016 (pp. 532-537). https://doi.org/10.1109/ICISCE.2016.120 
Idrees, S. M., Alam, M. A., \& Agarwal, P. (2018). A study of big data and its challenges. International Journal of Information Technology. https://doi.org/10.1007/s41870-018-0185-1

Justo, R., Corcoran, T., Lukin, S. M., Walker, M., \& Torres, M. I. (2014). Extracting relevant knowledge for the detection of sarcasm and nastiness in the social web. Knowledge-Based Systems, 69(1), $124-133$. https://doi.org/10.1016/j.knosys.2014.05.021

Kitchenham, B. (2004). Procedures for performing systematic reviews. Keele, UK, Keele University, 33(TR/SE-0401), 28. https://doi.org/10.1.1.122.3308

Kumar, A., \& Sachdeva, N. (2019). Cyberbullying detection on social multimedia using soft computing techniques: a meta-analysis. Multimedia Tools and Applications. https://doi.org/10.1007/s11042-019-7234-z

Langford, C. H. (1938). Morris Charles W.. Foundations of the theory of signs. International encyclopedia of unified science, vol. 1, no. 2. The University of Chicago Press, Chicago 1938, vii +59 pp. The Journal of Symbolic Logic, 3(04), 158. https://doi.org/10.2307/2267781

Levitan, S. I., An, G., Wang, M., Mendels, G., Hirschberg, J., Levine, M., \& Rosenberg, A. (2015). Cross-Cultural Production and Detection of Deception from Speech. In Proceedings of the 2015 ACM on Workshop on Multimodal Deception Detection (pp. 1-8). New York, NY, USA: ACM. https://doi.org/10.1145/2823465.2823468

Losada, D. E., \& Crestani, F. (2016). A Test Collection for Research on Depression and Language Use. In N. Fuhr, P. Quaresma, T. Gonçalves, B. Larsen, K. Balog, C. Macdonald, ... N. Ferro (Eds.) (Vol. 9822, pp. 28-39). Cham: Springer International Publishing. https://doi.org/10.1007/978-3-319-44564-9_3

Lundquist, D., Zhang, K., \& Ouksel, A. (2015). Ontology-driven cyber-security threat assessment based on sentiment analysis of network activity data. Proceedings - 2014 International Conference on Cloud and Autonomic Computing, ICCAC 2014, 5-14. https://doi.org/10.1109/ICCAC.2014.42

Maynard, D., Bontcheva, K., \& Augenstein, I. (2016). Natural Language Processing for the Semantic Web. Synthesis Lectures on the Semantic Web: Theory and Technology (Vol. 6). Morgan \& Claypool. https://doi.org/10.2200/s00741ed1v01y201611wbe015

Mundra, S., Mannarswamy, S., Sinha, M., \& Sen, A. (2017). Embedding Learning of Figurative Phrases for Emotion Classification in Micro-Blog Texts. In Proceedings of the Fourth ACM IKDD Conferences on Data Sciences (pp. 1-9). New York, NY, USA: ACM. https://doi.org/10.1145/3041823.3041828

Omar, S. J., Fred, K., \& Swaib, K. K. (2018). A state-of-the-art review of machine learning techniques for fraud detection research. In Proceedings of the 2018 International Conference on Software Engineering in Africa (pp. 11-19). New York, NY, USA: ACM. https://doi.org/10.1145/3195528.3195534

Pandey, R., Purohit, H., Stabile, B., \& Grant, A. (2018). Distributional Semantics Approach to Detect Intent in Twitter Conversations on Sexual Assaults. In 2018 IEEE/WIC/ACM International Conference on Web Intelligence (WI) (pp. 270-277). IEEE. https://doi.org/10.1109/WI.2018.00-80

Pandey, R., Purohit, H., Stabile, B., \& Grant, A. (2019). Distributional Semantics Approach to Detect Intent in Twitter Conversations on Sexual Assaults. Proceedings - 2018 IEEE/WIC/ACM International Conference on Web Intelligence, WI 2018, 270-277. https://doi.org/10.1109/WI.2018.00-80

Park, G., \& Rayz, J. (2019). Ontological Detection of Phishing Emails. Proceedings - 2018 IEEE International Conference on Systems, Man, and Cybernetics, SMC 2018, 2858-2863. https://doi.org/10.1109/SMC.2018.00486

Peirce, C. S. (1994). The Collected Papers of Charles S. Peirce, 1597.

Raisi, E., \& Huang, B. (2017). Cyberbullying Detection with Weakly Supervised Machine Learning. In Proceedings of the 2017 IEEE/ACM International Conference on Advances in Social Networks Analysis and Mining 2017 (pp. 409-416). New York, NY, USA: ACM. https://doi.org/10.1145/3110025.3110049

Ravi, K., \& Ravi, V. (2015). A survey on opinion mining and sentiment analysis: Tasks, approaches and applications. Knowledge-Based Systems, 89, 14-46. https://doi.org/10.1016/j.knosys.2015.06.015

Rosa, H., Pereira, N., Ribeiro, R., Ferreira, P. C., Carvalho, J. P., Oliveira, S., ... Trancoso, I. (2019). Automatic cyberbullying detection: A systematic review. Computers in Human Behavior, 93, 333-345. https://doi.org/10.1016/j.chb.2018.12.021

Salawu, S., He, Y., \& Lumsden, J. (2017). Approaches to Automated Detection of Cyberbullying: A Survey. IEEE Transactions on Affective Computing, 1. https://doi.org/10.1109/TAFFC.2017.2761757

Searle, J. R. (1969). Speech Acts: An Essay in the Philosophy of Language. Cambridge: Univ. Press. Retrieved from http://www.amazon.de/Speech-Acts-Essay-Philosophy-Language/dp/052109626X

Sharma, M., \& Sarma, K. K. (2017). Learning aided mood and dialect recognition using telephonic speech. 2016 International Conference on Accessibility to Digital World, ICADW 2016 - Proceedings, $163-167$. https://doi.org/10.1109/ICADW.2016.7942534

Suárez-Serrato, P., Velázquez Richards, E. I., \& Yazdani, M. (2018). Socialbots Supporting Human Rights. In Proceedings of the 2018 AAAI/ACM Conference on AI, Ethics, and Society - AIES '18 (pp. 290-296). New York, New York, USA: ACM Press. https://doi.org/10.1145/3278721.3278734 
Teh, P. L., Cheng, C.-B., \& Chee, W. M. (2018). Identifying and Categorising Profane Words in Hate Speech. In Proceedings of the 2Nd International Conference on Compute and Data Analysis (pp. 65-69). New York, NY, USA: ACM. https://doi.org/10.1145/3193077.3193078

Teodorescu, H. N., \& Saharia, N. (2015). An internet slang annotated dictionary and its use in assessing message attitude and sentiments. In 2015 International Conference on Speech Technology and Human-Computer Dialogue, SpeD 2015 (pp. 1-8). https://doi.org/10.1109/SPED.2015.7343084

Waseem, Z., Thorne, J., \& Bingel, J. (2018). Bridging the Gaps: Multi Task Learning for Domain Transfer of Hate Speech Detection. In J. Golbeck (Ed.), Online \{Harassment\} (pp. 29-55). Cham: Springer International Publishing. https://doi.org/10.1007/978-3-319-78583-7_3

Wu, L., Morstatter, F., \& Liu, H. (2018). SlangSD: building, expanding and using a sentiment dictionary of slang words for short-text sentiment classification. Language Resources and Evaluation, 52(3), 839-852. https://doi.org/10.1007/s10579-018-9416-0

Xiaomei, Z., Jing, Y., \& Jianpei, Z. (2018). Sentiment-based and hashtag-based Chinese online bursty event detection. Multimedia Tools and Applications, 77(16), 21725-21750. https://doi.org/10.1007/s11042-017-5531-y

Zhang, Z., Robinson, D., \& Tepper, J. (2018). Detecting Hate Speech on Twitter Using a Convolution-GRU Based Deep Neural Network. In Advances in Information Technologies for Electromagnetics (Vol. 1, pp. 745-760). Springer International Publishing. https://doi.org/10.1007/978-3-319-93417-4_48

\section{APÊNDICE I}

Tabela 1. Síntese dos Trabalhos Relacionados

\begin{tabular}{|c|c|c|c|c|c|c|c|c|c|c|c|}
\hline \multirow{2}{*}{ Referência } & \multicolumn{3}{|c|}{ Objetivo } & \multicolumn{8}{|c|}{ Aplicação } \\
\hline & $\mathbf{A}$ & $\mathbf{R}$ & D & 1 & 2 & 3 & 4 & 5 & 6 & 7 & 8 \\
\hline (Ravi \& Ravi, 2015) & $\mathrm{X}$ & & & & & $\mathrm{X}$ & $\mathrm{X}$ & & & $\mathrm{X}$ & \\
\hline (Salawu et al., 2017) & & & $\mathrm{X}$ & & $\mathrm{X}$ & & & & & & \\
\hline (Fortuna \& Nunes, 2018) & & & $\mathrm{X}$ & $\mathrm{X}$ & & & & & & & $\mathrm{X}$ \\
\hline (Omar et al., 2018) & & & $\mathrm{X}$ & & & & & & $\mathrm{X}$ & $\mathrm{X}$ & \\
\hline (Kumar \& Sachdeva, 2019) & & & $\mathrm{X}$ & & $\mathrm{X}$ & & & & & & $\mathrm{X}$ \\
\hline (Rosa et al., 2019) & & & $\mathrm{X}$ & & $\mathrm{X}$ & & & & & $\mathrm{X}$ & \\
\hline Este Estudo & $\boldsymbol{X}$ & $\boldsymbol{X}$ & $x$ & & & & & $\boldsymbol{X}$ & $\boldsymbol{X}$ & $x$ & $\boldsymbol{X}$ \\
\hline
\end{tabular}

Legenda: Objetivos: (A) Análise; (R) Representação/Formalização; e (D) Detecção. Aplicações: (1) Discurso de Ódio; (2) Cyberbullying; (3) Mineração de Opiniões; (4) Sentimentos; (5) Intenções; (6) Fraudes e Crimes; (7) Aprendizado de Máquina; e (8) Mídias Sociais.

\section{APÊNDICE II}

Tabela 2. Critérios de inclusão e exclusão de artigos

\begin{tabular}{c|cl}
\hline Tipo & Sigla & \multicolumn{1}{c}{ Critério } \\
\hline Inclusão & I1 & Pesquisas sobre análise de intenções em linguagem natural em redes sociais. \\
& I2 & Pesquisas sobre análise de linguagens cifradas (ex.: gírias). \\
& I3 & Estudos que utilizam a teoria dos atos de fala ou semiótica para análise de linguagens cifradas. \\
I4 & Estudos que utilizam ontologias para representação do conhecimento sobre atos criminosos. \\
Exclusão & E1 & Artigos escritos em idiomas diferentes do Inglês e do Português. \\
& E2 & Artigos que não estejam relacionados com análise de intenção ou emoção e pelo menos um dos seguintes temas: \\
& E3 & análise de linguagem cifrada, teoria dos atos da fala, ontologias, e semiótica. \\
& E4 & Textos que não sejam publicações científicas. \\
& E5 & Resumos com menos de 4 páginas e que não tenham profundidade ou resultados relevantes. \\
& E6 & Revisões sistemáticas e Livros. \\
\hline
\end{tabular}




\section{APÊNDICE III}

Tabela 3. Síntese dos trabalhos analisados

\begin{tabular}{|c|c|c|c|c|c|c|c|c|c|c|c|c|c|c|}
\hline \multirow[t]{2}{*}{ Autores } & \multicolumn{4}{|c|}{ Objetivos } & \multicolumn{10}{|c|}{ Aplicações } \\
\hline & A & $\mathbf{R}$ & D & $\mathbf{P}$ & 1 & 2 & 3 & 4 & 5 & 6 & 7 & 8 & 9 & 10 \\
\hline (Justo et al., 2014) & & & $\mathrm{X}$ & & & & & & $\mathrm{X}$ & & & $\mathrm{X}$ & & \\
\hline (Teodorescu \& Saharia, 2015) & & $\mathrm{X}$ & & & & & & $\mathrm{X}$ & & & & & $\mathrm{X}$ & \\
\hline (Levitan et al., 2015) & & & $\mathrm{X}$ & & & & & $\mathrm{X}$ & & & & & & \\
\hline (Appling et al., 2015) & & & & $\mathrm{X}$ & & & & $\mathrm{X}$ & & & & & & \\
\hline (Hagen et al., 2015) & $\mathrm{X}$ & & & & & & & & & $\mathrm{X}$ & & & & \\
\hline (Lundquist et al., 2015) & & $\mathrm{X}$ & & & & & & $\mathrm{X}$ & & & & & & $\mathrm{X}$ \\
\hline (Losada \& Crestani, 2016) & & $\mathrm{X}$ & & & & & & $\mathrm{X}$ & & & & & & \\
\hline (Chen et al., 2016) & $\mathrm{X}$ & & $\mathrm{X}$ & & & & & & $\mathrm{X}$ & & & & & \\
\hline (Dhouioui \& Akaichi, 2016) & & & $\mathrm{X}$ & & & & & & & $\mathrm{X}$ & & $\mathrm{X}$ & & \\
\hline (Hu \& Wang, 2016) & $\mathrm{X}$ & & & & & & & & & $\mathrm{X}$ & $\mathrm{X}$ & & & \\
\hline (Barreira et al., 2017) & $\mathrm{X}$ & & & & & & & & & & & & & $\mathrm{X}$ \\
\hline (Agarwal \& Sureka, 2017) & $\mathrm{X}$ & & & & & & & & $\mathrm{X}$ & $\mathrm{X}$ & & $\mathrm{X}$ & & \\
\hline (Raisi \& Huang, 2017) & & & $\mathrm{X}$ & & & $\mathrm{X}$ & & & & & $\mathrm{X}$ & & & \\
\hline (Escalante et al., 2017) & & & $\mathrm{X}$ & & & & & $\mathrm{X}$ & & & & & & \\
\hline (Mundra et al., 2017) & $\mathrm{X}$ & & & & & & & $\mathrm{X}$ & & & & $\mathrm{X}$ & & \\
\hline (Sharma \& Sarma, 2017) & & & $\mathrm{X}$ & & & & & $\mathrm{X}$ & & & & & $\mathrm{X}$ & \\
\hline (Aghababaei \& Makrehchi, 2016) & & & & $\mathrm{X}$ & & & & & & $\mathrm{X}$ & & $\mathrm{X}$ & & \\
\hline (García-Díaz et al., 2018) & $\mathrm{X}$ & & & & & & & $\mathrm{X}$ & & & $\mathrm{X}$ & & & \\
\hline (Anzovino et al., 2018) & & & $\mathrm{X}$ & & & & & & & $\mathrm{X}$ & & $\mathrm{X}$ & $\mathrm{X}$ & \\
\hline (Waseem et al., 2018) & & & $\mathrm{X}$ & & $\mathrm{X}$ & & & & & & & & & \\
\hline (Zhang et al., 2018) & & & $\mathrm{X}$ & & $\mathrm{X}$ & & & & & & $\mathrm{X}$ & $\mathrm{X}$ & & \\
\hline (Teh et al., 2018) & & & $\mathrm{X}$ & & $\mathrm{X}$ & & & & & & & & & \\
\hline (Ghosh et al., 2018) & $\mathrm{X}$ & & & & & & & & $\mathrm{X}$ & & & & & $\mathrm{X}$ \\
\hline (Xiaomei et al., 2018) & & & $\mathrm{X}$ & & & & & $\mathrm{X}$ & & & & & & $\mathrm{X}$ \\
\hline (Suárez-Serrato et al., 2018) & & $\mathrm{X}$ & & & & & & & $\mathrm{X}$ & & & $\mathrm{X}$ & & \\
\hline $\begin{array}{c}\text { (Pandey, Purohit, Stabile, \& } \\
\text { Grant, 2018) }\end{array}$ & & & $X$ & & & & & & $X$ & $X$ & & $X$ & & $X$ \\
\hline (Park \& Rayz, 2019) & & & $\mathrm{X}$ & & & & & & $\mathrm{X}$ & $\mathrm{X}$ & & $\mathrm{X}$ & & $\mathrm{X}$ \\
\hline
\end{tabular}

Legenda: Objetivos: (A) Análise; (R) Representação/Formalização; (D) Detecção; e (P) Predição. Aplicações:

(1) Discurso de Ódio; (2) Cyberbullying; (3) Mineração de Opiniões; (4) Sentimentos; (5) Intenções; (6) Fraudes e Crimes; (7) Aprendizado de Máquina; (8) Mídias Sociais; (9) Linguagem Cifrada ou Criminal; e (10) Semântica. 